ORIGINAL ARTICLE

\title{
Correlation between clinical diagnosis and arthroscopic findings of the shoulder
}

\section{A M Malhi, R Khan}

Postgrad Med J 2005;81:657-659. doi: 10.1136/pgmj.2004.030254

See end of article for authors' affiliations

.....

Correspondence to: Mr A M Malhi, 40

Wadham Gardens,

Greenford, Middlesex UB6 OBP, UK; amalhi@doctors. org.uk

Submitted

2 November 2004

Accepted 6 March 2005 Objective: To assess the accuracy of clinical examination by non-specialist orthopaedic surgeons of
patients presenting to a diagnostic and treatment centre (DTC) for arthroscopic shoulder surgery. Methods: A retrospective review of notes of 130 consecutive shoulder arthroscopies performed at a DTC over a 10 month period. Preoperative clinical diagnosis was compared with operative arthroscopic findings. Additional information from preoperative imaging was compared with clinical examination and arthroscopic findings. Preoperative clinical examinations and consent were undertaken by clinical fellows, (SpR level) and non-upper limb consultant orthopaedic surgeons. Consultants specialising in upper limb surgery performed the operations.

Results: Six main groups were identified on the basis of clinical examination: impingement 76 cases $(58 \%)$, instability 22 cases (17\%), frozen shoulder 11 cases $(8 \%)$, rotator cuff tear four cases $(3 \%)$, nonspecific pain eight cases (6\%), and normal clinical examination nine cases (7\%). Impingement and instability diagnosed clinically strongly correlated with the arthroscopic findings. Clinical diagnosis of frozen shoulder and rotator cuff tears had a weaker correlation with the arthroscopic findings. Of the nine cases of normal clinical examination, abnormality was found at arthroscopy in all cases.

Conclusion: There have been very few studies comparing clinical examination of the shoulder with arthroscopic findings. This study emphasises the importance of good clinical examination skills in diagnosing common shoulder abnormalities. The addition of imaging, particularly ultrasound and magnetic resonance imaging further increases the likelihood of an accurate diagnosis. Shoulder examination should be taught with as much emphasis at both undergraduate and postgraduate level as other orthopaedic clinical examinations.
A fter spinal and knee problems, shoulder complaints are the third most common cause of musculoskeletal problems in the UK. ${ }^{1}$ However, teaching of clinical examination of the shoulder is not something given as much emphasis as that of the knee, hip, and spine. The increasing sub-specialisation within orthopaedics also means that the opportunity to refine examination skills is limited unless working for an upper limb surgeon.

Our study compared preoperative diagnoses with arthroscopic findings at a diagnostic and treatment centre (DTC). There are very few studies comparing clinical examination with arthroscopic findings. ${ }^{23}$ In addition we also noted what imaging was available to the consenting surgeon in the preadmission clinic. Preoperative consent was based on the clinical findings and imaging present in the clinic. This was particularly important in the DTC setting as all of the patients were referred from other hospitals with most waiting for long periods for their surgery. Their clinical presentation may have changed since initially being listed. In addition an increasing number of patients are now directly referred to DTCs by primary care trusts, particular by GPs with a special interest in orthopaedics.

\section{METHODS}

A retrospective review of all shoulder arthroscopies between October 2002 and July 2003 (10 months) was undertaken. The preoperative diagnoses were obtained from the notes on the basis of the history and clinical examination and compared with the operative findings at arthroscopy. Imaging available at the pre-admission clinics was noted. Consent was taken by clinical fellows (specialist registrar level) and non-upper limb consultant surgeons.

\section{RESULTS}

The study population consisted of 72 men and 58 women with ages ranging from 19 to 85 years (average age 46.8).

Six main groups were identified on the basis of clinical examination: impingement 76 cases $(58 \%)$, instability 22 cases $(17 \%)$, frozen shoulder 11 cases $(9 \%)$, rotator cuff tear four cases $(3 \%)$, non-specific pain eight cases $(6 \%)$, and normal clinical examination nine cases $(7 \%)$.

\section{Impingement subgroup}

A clinical diagnosis of impingement was obtained from the notes by positive Hawkins' test, modified Neer test, painful arc, sub-acromial crepitus, or if stated simply as impingement positive.

At operation, presence of an impingement lesion, bursitis, or a sub-acromial spur was recorded.

Of the 76 cases of impingement diagnosed pre-operatively 63 cases were confirmed at arthroscopy (83\%). Thirteen cases were not confirmed at arthroscopy (17\%). This gives a sensitivity of 0.84 , positive predictive value (PPV) of 0.83 , a specificity of 0.76 , negative predictive value (NPV) of 0.78 . Of the 13 patients not confirmed to have impingement at arthroscopy there were various lesions visualised (table 1).

Of interest 12 cases of impingement were picked up arthroscopically that were not diagnosed clinically. Table 2 shows the preoperative diagnoses of these cases.

Of the 76 cases diagnosed preoperatively as impingement, 36 had some form of imaging available to the consenting surgeon in clinic. Fifteen patients had plain radiographs and

Abbreviations: DTC, diagnostic and treatment centre; MRI, magnetic resonance imaging; US, ultrasound; PPV, positive predictive value; NPV, negative predictive value 
Table 1 Operative findings in cases where impingement was diagnosed preoperatively

\begin{tabular}{ll}
\hline Operative findings & Number of cases \\
\hline Impingement & 63 (83\%) \\
Frozen shoulder & 3 \\
Supraspinatus tear & 1 \\
Abnormal ca ligament & 2 \\
Calcium deposit & 1 \\
Biceps rupture & 1 \\
Loose body & 1 \\
Degenerative change & 1 \\
Normal arthroscopy & 3 \\
\hline
\end{tabular}

21 had either magnetic resonance imaging (MRI) or ultrasound (US).

Of the 15 cases with plain radiographs, nine had abnormal radiographs suggesting impingement, for example, narrowing or bony spurs at the sub-acromial space. Of the six normal radiographs, five had impingement at arthroscopy. Plain radiography correctly identified impingement in $60 \%$ of cases compared with clinical examination, which identified $80 \%$ of cases preoperatively.

Of the 21 cases with MRI or US, apart from one false positive US, the imaging correctly identified all cases of impingement-that is, $95 \%$ of cases compared with the preoperative clinical examination, which correctly diagnosed $80 \%$ of cases.

Therefore clinical examination was superior to plain radiography but marginally inferior to MRI or US in diagnosing impingement.

\section{Instability subgroup}

A clinical diagnosis of instability was obtained from the notes by a positive apprehension or Jobe relocation test.

At arthroscopy the presence of a Hill Sachs, Bankart, or labral lesions was recorded.

Of the 22 cases clinically diagnosed 21 (96\%) were confirmed arthroscopically. This gives a sensitivity of 0.81 , PPV of 0.96 and a specificity of 0.01 , NPV of 0.95 . In one patient examination under anaesthesia and arthroscopy failed to identify instability (clinically false positive).

Conversely five cases reported as having a normal clinical examination were ultimately shown to have arthroscopic evidence of instability.

Nine cases had preoperative imaging available in the preadmission clinic. Four had plain radiographs and five had MRI scans.

Of the four plain films, three were normal and one was abnormal. Plain radiographs therefore only identified $25 \%$ of cases correctly.

Of the five MRI scans, two were normal and three were abnormal-that is, $60 \%$ of cases were correctly identified by MRI.

Clinical examination was more helpful in diagnosing instability than all forms of imaging.

\section{Frozen shoulder subgroup}

Clinical diagnosis of a frozen shoulder was obtained from the notes where there was a global reduction in range of movements with no other diagnosis offered.

At arthroscopy a contracted capsule or adhesive capsulitis was reported.

Of the 10 cases diagnosed clinically five (46\%) were confirmed arthroscopically. One case $(9 \%)$ was reported as a normal arthroscopy. Three cases (27\%) were reported as having impingement. Two cases ( $18 \%$ ) had rotator cuff tears.
Table 2 Preoperative clinical diagnosis where impingement was found at arthroscopy

\begin{tabular}{ll}
\hline Preoperative clinical diagnosis & Number of cases \\
\hline Frozen shoulder & 5 \\
Weak supraspinatus & 1 \\
Tender trapezius & 1 \\
Tender acromio-clavicular joint & 1 \\
Normal examination & 4 \\
\hline
\end{tabular}

This gives a sensitivity of 1, PPV of 0.46 and a specificity of 0.95, NPV of 1.

Only three cases had preoperative imaging available to the consenting doctor. Two plain radiographs that were reported as normal and ultimately were shown to be cases of impingement at operation. There was one MRI scan that was reported as abnormal but was not found to be a frozen shoulder at operation.

MRI and US scans were reported by radiologists. Most plain films were reported by radiologists but others commented on by the consenting surgeons.

\section{Rotator cuff tear subgroup}

Clinical diagnosis of a rotator cuff tear was obtained from the notes where there was reported weakness of supraspinatus or impairment of abduction.

Arthroscopy identified tears.

Four cases were clinically diagnosed. Three (75\%) were confirmed at arthroscopy. The remaining case was reported as a normal arthroscopy. This gives a sensitivity of $\mathrm{l}$ and a specificity of 0.99 .

All four cases had preoperative imaging available. Two had plain rdiographs, one had MRI, and the other had US. MRI and US both showed supraspinatus tears. Both radiographs showed degenerative changes.

\section{Normal clinical examination subgroup}

Nine patients were reported as having a normal clinical examination. Of these, five were found to have, under anaesthesia, arthroscopic evidence of instability. Four were found to have arthroscopic evidence of impingement.

\section{DISCUSSION}

Clinical diagnosis of impingement and instability correlated fairly well with the arthroscopic findings, $83 \%$ and $96 \%$ respectively of cases. As expected clinical examination was more helpful than plain radiographs for picking up both impingement and instability. MRI and US were superior to clinical examination in picking up impingement but not so for instability.

The rotator cuff tear subgroup also correlated well with operative findings. MRI and US were useful preoperatively. However, the correlation between blanket clinical diagnosis of a frozen shoulder and the operative findings did not correlate so well. However, the sample sizes for these two groups are small so may not be truly representative.

There have been very few studies comparing clinical shoulder examination with arthroscopic findings. A previous study by Norregaard et $a^{2}$ has shown a poor correlation between clinical tests for impingement, muscle tenderness, and weakness and labral tears with both US and arthroscopic findings. Our study has looked at the clinical findings of nonshoulder specialists. Our findings would suggest that instability and impingement are diagnoses that have physical signs that are easily elicited and as such have a high accuracy in preoperative diagnosis. Norregaard alludes to this fact and concludes that there would probably be a greater correlation 
in patients with traumatic shoulder instability. Another study by Ure et $\mathrm{al}^{3}$ had comparable findings to our study in that impingement was correctly diagnosed in 19 of 22 cases (86\%) and rotator cuff tears in seven of nine cases $(78 \%)$.

Orthopaedic clinical examination is a skill that is taught at undergraduate level and refined during postgraduate training. Emphasis, particularly at undergraduate level, is placed on hip, knee, and back examination. Shoulder examination is usually a skill gained while working with an orthopaedic team. With increasing sub-specialisation of orthopaedics, unless working with an upper limb surgeon, the scope for refining clinical skills in shoulder examination is limited. In addition, the increasing trend for GPs with specialist interests may mean that many direct referrals to orthopaedic surgeons for surgery will become more commonplace. It will therefore be important that not only trainee orthopaedic surgeons but also GPs have a firm grasp of orthopaedic clinical examination, particularly of the shoulder, as this is one of the commonest sites for musculoskeletal problems. ${ }^{1}$

This study reinforces the importance of a good clinical examination of the shoulder in making a most probable diagnosis particularly for impingement and instability. This is helpful to explain to the patient when taking informed consent for operative procedures. We feel that proper understanding and acquisition of appropriate clinical examination skills by non-specialist orthopaedic surgeons may improve diagnosis and patient management at both primary and hospital care level.

\section{Authors' affiliations}

A M Malhi, R Khan, Department of Orthopaedics, Ravenscourt Park Hospital, Hammersmith Hospitals NHS Trust, London, UK

Funding: none.

Conflicts of interest: none.

\section{REFERENCES}

1 Urwin M, Symmons D, Allison T, et al. Estimating the burden of musculoskeletal disorders in the community: the comparative prevalence of symptoms at different anatomical sites, and the relation to social deprivation. Ann Rheum Dis 1998;57:649-55.

2 Norregaard J, Krogsgaard MR, Lorenzen T, et al. Diagnosisng patients with longstanding shoulder joint pain. Ann Rheum Dis 2002:61:646-50.

3 Ure BM, Tiling T, Kirchner R, et al. Reliability of clinical examination of the shoulder in comparison with arthroscopy. A prospective study. Unfallchirurg 1993;96:328-6. afety of the medicines and equipment used in the treatment of patients is of paramount importance. This month's web trawl discusses UK based web sites dedicated to providing up to date information relating to such safety issues.

http://www.mhra.gov.uk This is the web site of the Medicines and Healthcare Regulatory Agency (MHRA); the agency of the Department of Health that protects the public by ensuring that all medicines and medical devices used in the UK are of an appropriate standard of safety and effectiveness. The MHRA is an amalgamation of two previously existing agencies, the Medical Devices Agency (MDA) and the Medicines Control Agency (MCA). From a common home page, the user is able to access current news items about medicines and devices, as well as the home pages of the two previously separate organisations. Clicking on the link for the "Medicines" brings the user to this section's home page (http://medicines.mhra.gov.uk). This is clearly laid out, is easy to use, and provides links to further pages detailing the work of the agency, its publications, and information resources. There is also a link to the Committee on Safety of Medicines, which is discussed further below. If instead, the user selects the link for the "Devices" section, they will be brought to the home page of the former Medical Devices Agency (http://devices.mhra. gov.uk). The colour scheme and layout of this section is very different to that of the "Medicines" section, suggesting that the two web sites may have been simply linked together when the agencies amalgamated. The layout of this page, is, perhaps, a little "busy", but nevertheless provides access to a wealth of news and information about the safety of medical devices. The layout does mean that it is somewhat difficult to navigate between pages - it can be difficult to find the way back to the home page- but nevertheless all the necessary links are there. Both of these web sites are updated regularly, and the "news" section seems to be updated on almost a daily basis. The web sites can be accessed without restriction by healthcare professionals and the public alike, although much of the information available is probably only of interest to those working within healthcare services in the UK.

http://medicines.mhra.gov.uk/aboutagency/regframework/csm/csmhomemain.htm This is the web site of the Committee on Safety of Medicines (CSM). Although it is accessed via the MHRA web site, it is in fact a separate entity, and once again of entirely different design to the MHRA's two component sites. From the home page, the user is directed to pages detailing the work and publications of the CSM, and it also provides a link to the UK's "yellow card scheme" for reporting adverse drug reactions. These can now be completed electronically. Detailed information on the scheme is provided that is aimed both at health professionals and members of the public, both being able to complete a yellow card if appropriate. Again access to the site is unrestricted, and the content will be of interest to anyone with a specific interest in drug related adverse events.

Robyn Webber

Web Editor 